\title{
Are 'Behavioural' Disorders a separate nosological category of disorders ?
}

\author{
Shrirang Bakhle \\ ${ }^{1}$ Consulting Physician, Mumbai. \\ E-mail-ss.bakhle@gmail.com
}

\begin{abstract}
The term 'Behavioural' Disorders is widely used in Psychiatry. For example, in the ICD-10, Chapter V is titled 'Mental and Behavioural Disorders'. There is a lack of consensus about the meaning of the term. Different organizations and persons use the term in different sense. This article discusses the meaning of the term and whether there is a separate nosological category of disorders called Behavioural Disorders. This article shows how there is no well-defined nosological category of disorders called Behavioural Disorders. BDs are not a nosological subcategory of Mental Disorders. There are no clear inclusion or exclusion criteria - in terms of behavioural abnormalities, interpersonal / social difficulties or functional impairment - that can be used to term a disorder as a BD or to exclude a disorder from the category of BDs. The behavior is not an organ of the body that we should create a separate nosological category of disorders for it. The use of the term Behavioural Disorders in double terms such as 'Mental and Behavioural Disorders' does not have any rationale. If the purpose of a nosology is to introduce clarity in categorization, then the use of the term Behavioural Disorders - especially as a double term - defeats the purpose. The rational description is to say 'Mental Disorders and their behavioural manifestations'. It is important to understand the conceptual difference between patients having 'disordered behavior' as a result of their Mental Disorders and terming them as Behavioural disorders.
\end{abstract}

Keywords: Behavioural Disorders, ICD-10, Psychiatric Nosology, Mental Disorders,

\section{INTRODUCTION}

The term 'Behavioural' Disorders is widely used in Psychiatry. For example, in the ICD-10, Chapter V is titled 'Mental and Behavioural Disorders'. Discussions with Psychiatrists around the world and a survey of literature revealed that there is no precise definition of the term. There is a lack of consensus about the meaning of the term. Different organizations and persons use the term in different sense. This article discusses the meaning of the term and whether there is a separate nosological category of disorders called Behavioural Disorders. 


\section{What is the meaning of 'Behavioural' Disorders?}

Should all disorders involving abnormal or altered behaviour be called Behavioural Disorders (BDs)? Consider Orthopaedic Disorders. Some of the orthopaedic disorders result in gross changes in the 'behaviour' of the patients: abnormal facies, gait, movements and bladder / bowel habits. Can we call such disorders as BDs? The answer is negative. We call a disorder a BD when the primary dysfunction is 'mental' with resultant 'behavioural' changes. Thus BDs are basically Mental Disorders with behavioural manifestations. Even if many Orthopaedic Disorders result in abnormal behaviour, we do not call them BDs.

\section{Is there any organ in the body called 'behaviour'?}

Is 'behaviour' an organ of the body that we should create a separate nosological category of disorders called BDs? Consider Hemiplegia due to a cerebrovascular event. Although the resultant external manifestations involve an upper and lower limb, do we categorize it as Upper and Lower Limb Disorder - or as a Brain Disorder? We know that although the external clinical manifestations involve upper and lower limb, the dysfunction is in the brain. Hence we categorize Hemiplegia as a brain disorder. In BDs, the behavioural changes are only the externally observable manifestations caused by mental dysfunctions. Hence calling such behavioural changes as BDs is incorrect.

The importance of recognizing the difference between dysfunctions and external clinical manifestations is best exemplified by the comparison of two disorders: [1] Hemiplegia due to a vascular event. [2] Conversion Disorder with paralysis. In both these disorders, the external manifestation is paralysis. Yet because of the different dysfunctions, hemiplegia is categorized as a Neurological Disorder while Conversion Disorder is categorized as a Mental Disorder. If we consider every 'altered or abnormal behavior' as a Behavioural Disorder, both these disorders would be classified as BDs. 'Behaviour' is not an organ of the body that we should create a nosological category for it.

\section{Multiple confusions created by the term 'Behavioural Disorders' (BDs)}

There are two types of confusions created by the term BDs. One type of confusion is about which disorders should be called BDs and which disorders should not be called BDs. The second confusion is in the use of the term BDs as a part of double terms such as 'Mental and Behavioural Disorders', 'Psychiatric and Behavioural Disorders', 'Emotional and Behavioural Disorders'.

\section{Which disorders should be called BDs and which should not be called BDs?}

Different clinicians and researchers include different disorders under the title of BDs. For example, NIH's MedlinePlus website says, "Child Behavior Disorders- also called Conduct Disorders". [1]

Others have included different permutations and combinations:

- "Oppositional Defiant Disorder, Conduct Disorder, Intermittent Explosive Disorder, Kleptomania, Pyromania and others". [2]

- "Adjustment disorders, Psychiatric disorders, personality changes". The paper further mentions, "...severe behavioural disorders, in particular depressive disorders ..." [3] 
- "...than those in which choices are essential (behavioural disorders)". The paper includes substance use disorders and anorexia nervosa as BDs. [4]

- "... of 12 major behavioural disorders, including delusions, hallucinations, aggression/agitation, depression, anxiety, elation/euphoria, apathy, disinhibition, irritability/lability, aberrant behaviour, sleep disturbances and eating disorders."[5]

- "Anxiety disorders, Disruptive behavioural disorders, Dissociative disorders, Emotional disorders, Pervasive developmental disorders".[6]

- "Social Anxiety Disorder (Social Phobia), Post-Traumatic Stress Disorder -PTSD, REM Sleep Behaviour Disorder, Obsessive Compulsive Disorder-OCD, Bipolar Disorder, Borderline Personality Disorder - BPD, Oppositional Defiant Disorder ODD, Attention Deficit Hyperactivity Disorder, - ADHD, Attention Deficit Disorder - ADD, Intermittent Explosive Disorder - IED, Reactive Attachment Disorder RAD, Passive-Aggressive Personality Disorder, Trichotillomania". [7]

Thus it can be observed that different persons or organizations consider different disorders as BDs. This is because there is no standard definition of BDs. There are no standard inclusion and exclusion criteria that determine why a disorder should be termed a BD or why it should not. Because of the vagueness of the term BDs, different Mental Health Professionals list different permutations and combinations of Mental Disorders as BDs.

\section{Attempting to create a definition of BDs}

Is it possible to define specific inclusion and exclusion criteria that can guide the inclusion and exclusion of disorders from a nosological category of 'BDs'. In order to do this, we also have to define what the 'Non-Behavioural Disorders' are. Can we attempt to define the inclusion and exclusion criteria in terms of behavioural abnormalities, interpersonal / social difficulties or functional impairment? It will not be successful because most of the Mental Disorders (such as Schizophrenia or Depression) and many of the non-mental disorders (such as the orthopaedic disorders) have these features. Hence it is not possible to define a separate category of disorders called BDs.

\section{Can a single Mental Disorder be sometimes a Behavioural Disorder and sometimes Non-Behavioural Disorder?}

Consider patients with Depression. Some of the patients have sad affect / behaviour. So can we term them as having Behavioural Disorder? On the other hand, some of the patients have sadness of mood but are able to maintain a normal affect / behavior. So we will not term them as having a Behavioural Disorder. This demonstrates the conceptual difference between 'disordered behaviour' and 'Behavioural Disorder'. Many patients with Mental Disorders have disordered behavior. But terming these disorders as Behavioural Disorders is illogical.

\section{Is there any rationale behind the use of double terms that include Behavioural Disorders?}

Consider ICD-10. The title of Chapter V is 'Mental and Behavioural Disorders'. Similarly in literature we can find double terms that include BDs: 'Psychiatric and Behavioural 
Disorders' [8], Emotional and Behavioural Disorders [9], Emotional/Behavioural Disorders [10].

Is it rational to use such double terms? Does it mean that there some Mental Disorders (MDs) and there are some other Behavioural Disorders (BDs) and that this chapter includes these two mutually exclusive sets of disorders?

Or does the use of two terms mean that two qualifiers are needed to define the disorders. That is, there are some MDs and some BDs. But this chapter describes a third set of disorders that are both mental and behavioural?

Or even the assumption that BDs are a subcategory of MDs does not provide the rationale to use the double terms. First, if the BDs are supposed to be a subcategory of MDs, then the title should be 'Mental Disorders (including Behavioural Disorders)'. Secondly, as described earlier, most of the MDs have behavioural manifestations (and so also many non-mental disorders). Thus we cannot define a separate subcategory of MDs that can be called BDs.

\section{Conclusion}

There is no well-defined nosological category of disorders called Behavioural Disorders. BDs are not a nosological subcategory of Mental Disorders. There are no clear inclusion or exclusion criteria that can be used to term a disorder as a BD or to exclude a disorder from the category of BDs. It is not possible to define inclusion and exclusion criteria for BDs in terms of behavioural abnormalities, interpersonal / social difficulties or functional impairment, since most of the MDs have such manifestations. The behavior is not an organ of the body that we should create a separate nosological category of disorders for it. The use of the term Behavioural Disorders in double terms such as 'Mental and Behavioural Disorders' does not have any rationale. Such a use does not add any value but only leads to unnecessary and avoidable confusion. If the purpose of a nosology is to introduce clarity in categorization, then the use of the term Behavioural Disorders especially as a double term - defeats the purpose. The rational description is to say 'Mental Disorders and their behavioural manifestations'. It is important to understand the conceptual difference between patients having 'disordered behavior' as a result of their Mental Disorders and terming them as Behavioural disorders. Hence the ICD-10, Chapter $V$ should be titled 'Mental Disorders' and not 'Mental and Behavioural Disorders'. As the leader in nosology worldwide, the forthcoming ICD-11 should discontinue and discourage the use of the term Behavioural Disorders.

\section{REFERENCES}

1. Child Behaviour Disorders; MedlinePlus, U.S. National Library of medicine website, accessed 2015 May 15. http://www.nil.nih.gov/medlineplus/childbehaviourdisorders.html

2. Behavioural Disorders; Kelty Mental Health website, accessed 2015 May 15, URLhttp://keltymentalhealth.ca/mental-health/disorders/behavioural-disorders.

3. Houeto JL, Mesnage V, Mallet L, Pillon B, Gargiulo M, du Moncel ST, Agid Y. Behavioural disorders, Parkinson's disease and subthalamic stimulation. J Neurol Neurosurg Psychiatry 2002;72(6):701-7.

4. Bienvenu OJ, Davydow DS, Kendler KS. Psyhciatric disorders versus behavioural disorders and the degree of genetic influence. Psychol Med 2011;41(1):33-40. 
5. Rosen HJ, Allison SC, Schauer GF, Gorno-Tempini ML, Weiner MW, Miller BL. Neuroanatomical correlates of behavioural disorders in dementia. Brain 2005;128(11)"2612-25.

6. Behavioural Disorder Symptoms, Causes and Effects. PsychGuides.com, accessed 2015 May 15. URL- http:// http://www.psychguides.com/guides/behavioural-disordersymptoms-causes-and-effects/

7. Behaviour Disorders. BehaviourDisorder.org, accessed 2015 May 17, URLwww.behaviourdisorder.org

8. Bouras N, Holt G. Psychiatric and Behavioural Disorders in Intellectual and Devellopmental Disabilities. Cambridge University Press, UK ; 2007.

9. Barkmann C, Schulte-Markwort M. Prevalence of emotional and behavioural disorders in German children and adolescents: a meta-analysis. J Epidemiol Comm Health 2012;66(3):194-203.

10. Definitions, characteristics and related information: Information about Emotional/Behavioural Disorders. Council for Children with Behavioural Disorders website, accessed on 2015 May 17, URL- http://www.ccbd.net/about/new-item

Acknowledgements - Nil.

Conflict of Interest - Nil.

Funding - Nil. 